【Technical Paper】

\title{
Development of Microfibril Absorbent Substantial for Hygiene Products using Compostable Agro-Residuals
}

\author{
Indumathi $\mathrm{TR}^{* 1}$ and Divya $\mathrm{R}^{* 2, \#}$ \\ ${ }^{* 1}$ Department of Costume design and Fashion, PSG College of Arts and Science, Coimbatore - 641048, Tamil Nadu, India. \\ ${ }^{* 2}$ Department of Costume design and Fashion, PSG College of Arts and Science, Coimbatore - 641014, Tamil Nadu, India
}

\begin{abstract}
An absorbent core structure is revealed for acquiring and absorbing aqueous-based liquids in hygiene products. The core is made of fiber pulp for wicking and absorbing an aqueous-based liquid and retaining the liquid against moderate pressure. Non-wood based regenerated cellulose fibers are an attractive alternative for cotton and wood pulp. The bast fibres and grass fibers are converted to pulp for its applicability as high absorbency, biodegradability and dissolute compost ability need for healthcare products. Enhancing the moisture transport and storage of the liquid by the delignified fibrils made serves more when compared to other natural fibers. The fibers are delignified by kraft pulping process for its strength, bulk, porosity and other functional advantages. Absorbent core properties from two different fiber pulps were investigated pertained good result. Fiber dimension and morphology were evaluated through SEM analysis and free swell test.
\end{abstract}

(Received 23 March, 2021; Accepted 11 September, 2021)

\section{Introduction}

The present study relays to fluid-absorbing core material for hygiene products, which typically include baby diapers, adult incontinence garments, and feminine hygiene pads are settled with agro residual fiber pulp material. Each of these hygiene products typically includes an absorbent core for absorbing and containing body fluids, with the cores being structurally combined with other elements that relate to the odor control, comfort and similar functions of the absorbent item.

In almost every case, the hygiene products should enviably perform several allied functions like uptake of fluids, typically referred to as "acquisition' or "uptake, should have the capacity to hold the liquid taken up, a property referred to as "absorbency". Finally, the structure should have the capacity to maintain the liquid for a desired period of time and against moderate pressure, referred to as "retention" [1]. This property is assisted by natural wood and nonwood cellulosic fibers, which are also provide consumer appeal in products such as sanitary napkins, incontinence pads, paper towels, facial wipes and tissues, etc. Recently the interest for renewable resources for fibres particularly of plant origin is increasing. Flax, hemp, jute, ramie, sisal, bamboo and coir are cellulosic fibers mainly used for technical purposes. Natural cellulose fibres have successfully proven their qualities on an ecological view of fibre materials [2-3]. Different cellulose fibres can be used for textile and technical applications like Protech, Mobiletech, Meditech, etc. e.g. leaf fibers, bast or stem fibres, Seed fibers and grass fibers. There is a huge potential to use agricultural waste such as sugarcane baggasse, Kenaf, Maize, Rice straw, etc., to generate significant contribution in hygiene product as absorbent pulp substance comparison to other synthetic regenerated cellulose fibers [2]. Fluff or fluff pulp is the term used to describe cellulose fibers which is cooking inside a digester to separate the fibres from the lignin, where as it is a natural glue-like substance that binds them together and these are typically incorporated to provide absorbency and some wicking property [3]. During this kraft pulping process at a certain temperature and pressure with added additives the lignin compound in the natural fibre is removed and completely done in with the

\# corresponding author: Divya R (Email: divyasathyam25@gmail.com) 
screening process. These kraft pulps are distinctively a high-strength type of pulp material includes porosity, bulk and other functional adventages.

The fibres selected for this pulping process is Hibiscus Sabdariffa.L (Bast fibre) and Saccharum Officinarum (Grass fibre). Hibiscus Sabdariffa.L (Roselle) is a unique species cultivated in many tropical regions for its leaves, seeds, stem and calyces. Roselle has drawn the attention of food, beverage and pharmaceutical manufacturers because of its commercial potential as a natural food and colouring agent that can replace some synthetic products. It is considered as one of the important medicinal plant in some parts of the world. The extractions of Roselle have been used medicinally to treat colds, urinary tract infections and hangovers. Ethno botanical information of Roselle plant revealed diuretic, uricosuric, antibacterial, antifungal agent, sedative, antihypertensive, kidney stone treatment, liver damage treatment, etc. The bast fibres, and sometimes the whole stem, have been used in the paper industry in the United States and Asia [4]. Saccharum Officinarum (Sugarcane) is a Poaceae commonly cultivated in tropical areas. Cane stalk is crushed in sugar mills, generating $30 \%$ of residue left after crushing called bagasse. Bagasse comes from different parts of the cane stalk comprising the outside rind crushed with the inner pith. It contains $45 \%$ of fiber and composed of $45 \%$ cellulose, 33\% hemicelluloses, and 20\% lignin [5]. As bagasse is a fibrous residues which are either thrown into the wild as waste or used as fuel in the sugar plants as heat source. However, because of its high fiber content and particularly because of its cellulose rate, bagasse can be used to produce sustainable fibers. The bagasse fiber of sugar cane has direct application in ecomaterials [6].

\section{Meterials and Methods}

\subsection{Selection of fibers}

H. Sabdariffa and Saccharum Officinarum Stem which was grown in warm and humid tropical climate as the temperature ranges within 18 to $35{ }^{\circ} \mathrm{C}$ were collected from a farmer of Karnataka (Fig. 1). Harvested stems of 2 meter long are steeped in water for two weeks, followed by shedding of the bark and subsequently the stems are beaten to separate their fibres. The beaten stems are washed, dried and cut to a length of one inches for pulping process. The Saccharum Officinarum bagasses were collected from Tamil Nadu Newsprint limited, Karur, India.

\subsection{Pulping process}

We make kraft pulp by mixing non-wood fibres with a solution of alkali and water, and cooking them inside a digester. This separates the fibres from the lignin, which is a natural glue-like substance that binds them together. A rotating digester is a large tank, inside it, a combination of water black liquior, heat and pressure dissolve the lignin and begin the process of converting fiber to pulp [7]. From the digester, the cooked fibre is transferred into a shredding tank, where the delignified fiber to separate into individual fibrils. The pulp is then screened to remove any fibre bundles that have failed to separate and washed thoroughly to remove chemicals and dissolved lignin (Fig. 2).

H. Sabdariffa fiber is taken as $500 \mathrm{gms}$ of oven dry weight with bath ratio 1:4, liquor $15 \%$ and water $590 \mathrm{ml}$.The cooking temperature will be $165{ }^{\circ} \mathrm{C}$. After the fiber was cooked, it was shredded, and washed. The washed pulp was screened with $0.25 \mathrm{~mm}$ scale, as the rejects results of .1 to $.2 \%$. Finally the pulp was dried and exhausted to fluff pulp. The Saccharum Officinarum bagasses is taken as 500 gms of oven dry weight with bath ratio 1:4, liquor $15 \%$ and water 600

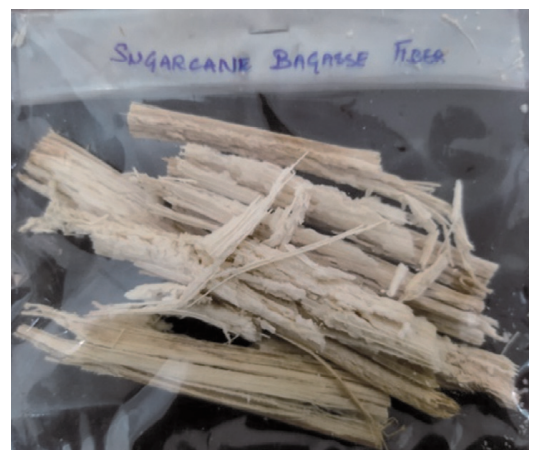

(b)

Fig. 1 (a) H. Sabdariffa Stem Fiber, (b) Saccharum Officinarum bagasses Fiber 


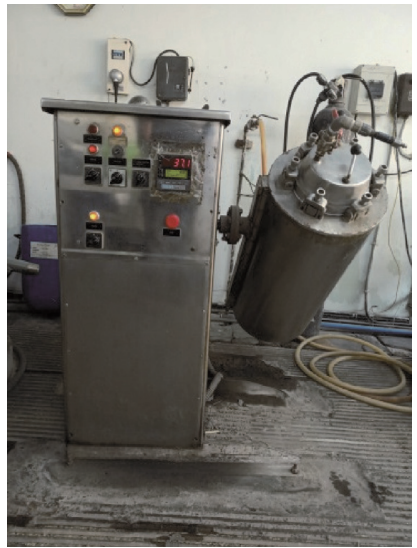

(a)

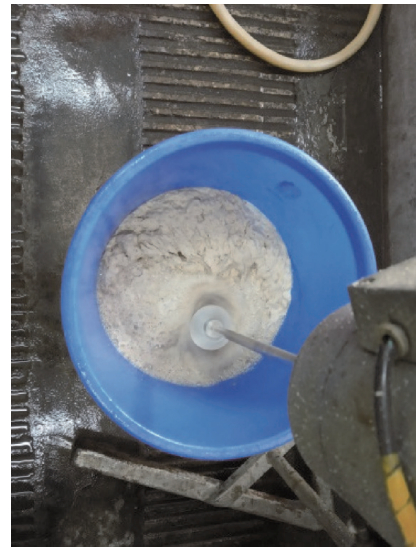

(b)

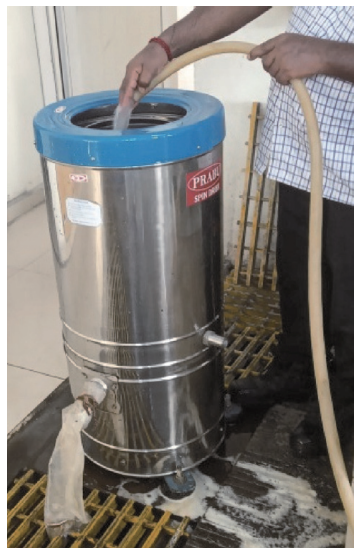

(c)

Fig. 2 (a) Rotating Digester for pulping, (b) Shredding, (c) Washing and Drying in Drier

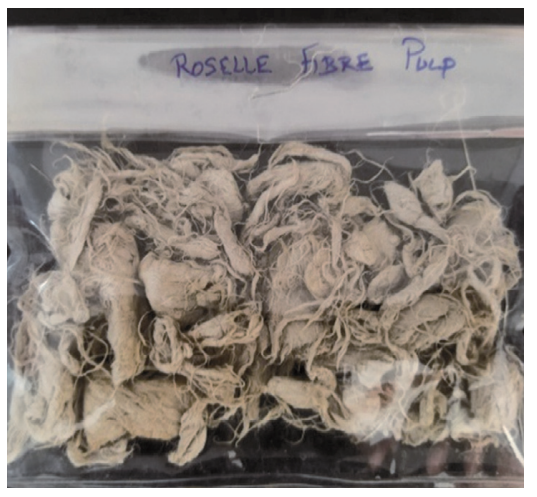

(a)

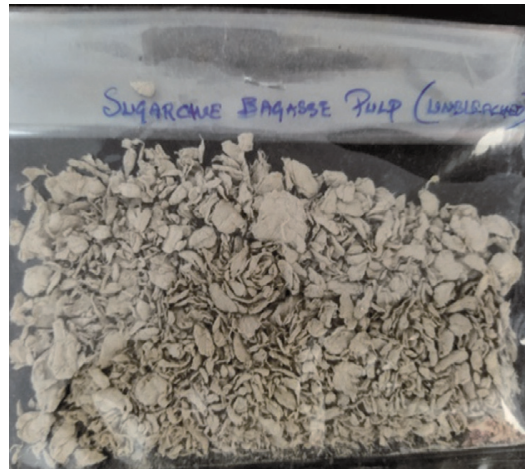

(b)

Fig. 3 (a) Hibiscus Sabdariffa stem fiber pulp, (b) Saccharum Officinarum bagasses fiber pulp

$\mathrm{ml}$. The fiber was cooked at the temperature of $160^{\circ} \mathrm{C}$, shredded, and washed. The washed pulp was screened with $0.25 \mathrm{~mm}$ scale, as the rejects results of 0 to $.1 \%$. Finally the pulp was dried and exhausted to fluff pulp (Fig. 3).

\subsection{Field Emission Scanning Electron Microscope Analysis}

Ultra 55 FE- SEM is general purpose ultra-high resolution equipment, was used in this study to capture images of fiber sample. The SEM micro graphs are taken on Zeiss field emission scanning electron microscopes at different magnifications of bast fibers and fibrils (pulp) to characterize their surface properties. Before the SEM analysis the samples were coated with gold (AU/C) for good conductivity and quality image. The image was captured at a scale of $20 \mu \mathrm{m}$ (varying magnifications) at high energy potential of $10000 \mathrm{KV}$. The distance between the probe and the sample stub was adjusted at different levels keeping in view the image clarity and its sharpness. The samples were scanned under different magnification levels ranging from 100X to 3000X [8].

\subsection{Free-Swell Test}

This method is the most conventional, fast, and suitable for limited amounts of samples ( $\left.\mathrm{w}_{0}=0.1-0.3 \mathrm{~g}\right)$. The core sample is placed into a tea-bag (acrylic/ polyester gauze with fine meshes) and the bag is dipped in an excess amount of water or saline solution for one hour to reach the equilibrium swelling. Then excess solution is removed by hanging the bag until no liquid is dropped off. The tea bag is weighed $\left(\mathrm{W}_{1}\right)$ and the swelling capacity is calculated by equation. The method's precision has been determined to be around $\pm 3.5 \%$. Here we analyze the quantities of the vital affiliations between the fibre structure and water, were they determined the size of capillaries in the fibre cells and water distribution in the fibre structure.

\section{Results and Discussions}

\subsection{Evaluation of Adsorbent Core Material}

The Cellulosic fiber and pulp samples were subjected to microscopic analysis for observing morphological variations and also for free swell test to evaluate the absorbency efficacy. 


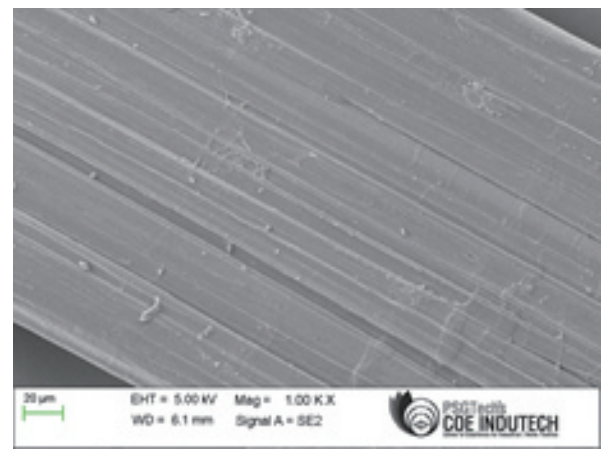

(a)

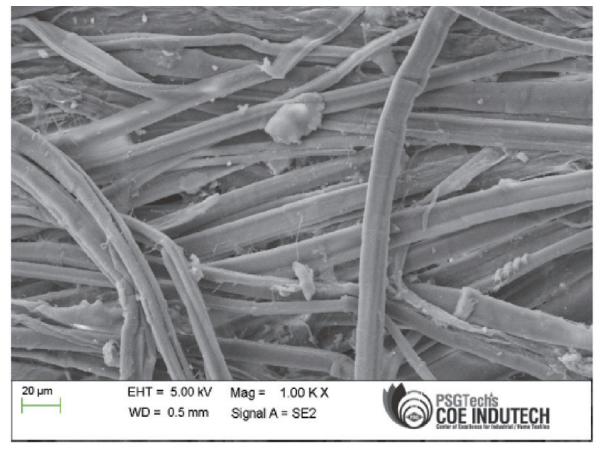

(b)

Fig. 4 SEM analysis of Sabdariffa Stem fiber (a) and fibrils(pulp) (b)

\subsubsection{Micro structural Study of Core (Cellulosic) Substantial}

Field emission scanning electron microscope was used to capture images of fiber and fibril material samples coated with AU/C to examine the differential morphology of cellulosic fiber and pulp fibrils (Fig. 4).

In this, the Sabdariffa stem fiber (a) and fibril (pulp) (b) specimen is magnified as $20 \mu \mathrm{m}$ to analyze the fixed specimen structure. The Fig. 4 (a) shows the sample cellulose fiber bonded with lignin and the Fig. 4 (b) shows the cellulose fibrils disintegrated and identified with lumen structure characterized by high porous, due to the removal of lignin during cooking with white liquor and water at certain temperature and pressure concern as pulping process. During this nearly $75 \%$ of lignin is removed and remaining under screening process, creates high porosity leads to increase in liquid absorption.

Field emission scanning electron microscope was used in this study to capture images of fiber and fibril material samples. In this, the Saccharum Officinarum stem fiber of Fig. 5 (a) and fibril specimen of Fig. 5 (b) is magnified as $20 \mu \mathrm{m}$ to analyze the fixed specimen structure which was found as several folded layers with high porous structure tends to absorb more liquid and swells with the molecules as fibrils at the pulp stage. The of Fig. 5 (a) shows the sample cellulose fiber bonded with lignin and the of Fig. 5 (b) shows the cellulose fibrils isolated and identified with significant diameter, characterized by high porous, due to the removal of lignin during cooking with white liquor and water at certain temperature and pressure concern as pulping process. During this nearly $75 \%$ of lignin is removed and remaining under screening process, modifies the fibre structure leads to increase in liquid absorption.

\subsection{Free Swell Test}

Free swelling is the effect of liquid sorption by cellulose fibrils to determine the liquid penetration and quantity with holding capability. The cellulosic fiber pulp for center core of the hygiene product was tested under standard ISO: 17190-5 for its swelling capacity. The fibers were weighted initially as 5 grams and optimal time and level of sorption is noted. This was done to determine the absorption level of the Sabdariffa and Saccharum Officinarum fibril pulp present as the core, by tea bag method in the laboratory of SITRA as valued result is given in the Table 1.

The test result shows that the absorbent layer (Sabdariffa and Saccharum Officinarum) obtains excellent absorbing capacity of nearly $60-70 \%$, to

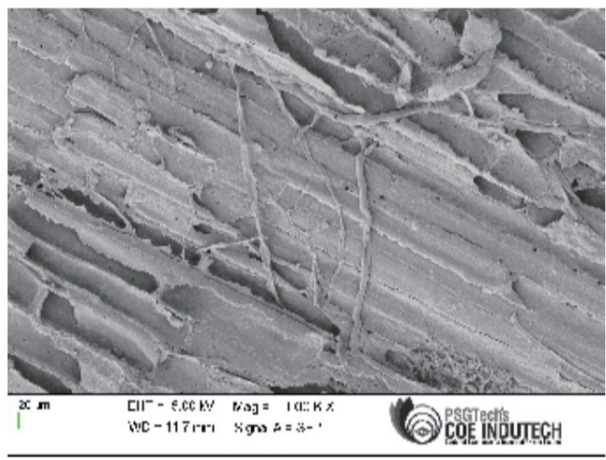

(a)

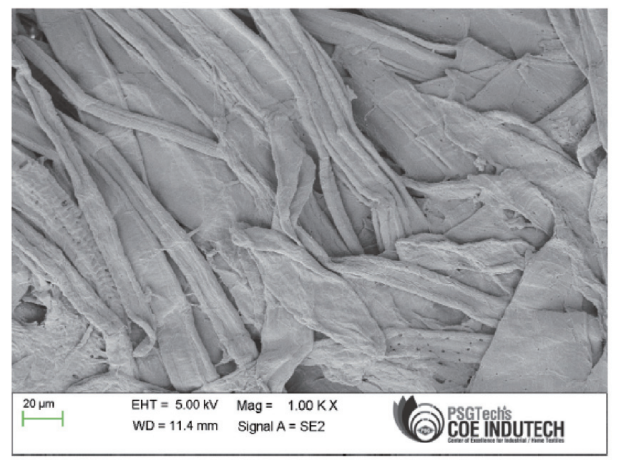

(b)

Fig. 5 SEM analysis of Saccharum Officinarum Stem fiber (a) and fibrils (pulp) (b) 
Table 1 Assessment of swelling capacity of core layer

\begin{tabular}{|c|c|}
\hline Free swell absorptive capacity & Core (Grams/Gram) \\
\hline Sabdariffa fiber pulp & 10.01 \\
\hline Saccharum Officinarum fiber pulp & 10.70 \\
\hline
\end{tabular}

serve the product as core layer and retain the fluid for long time. Hence these agro-residual pulps will assist as excellent absorbent material for health care and hygiene products.

\section{Conclusion}

Consumer experiences of disposable hygiene product materials designed and developed expensively by the manmade elements in market are replaced by the natural agricultural by products and wastes are focused in this study pertained good result. The invention is an absorbent core structure for acquiring and absorbing aqueous-based liquids in hygiene products. The core structure comprises a macroscopically homogeneous fluff pulp for wicking, absorbing aqueous-based liquids and retaining the liquid against moderate pressure is evaluated. Natural agro residual waste materials as core are playing major role through its diversified applications and undoubtedly the future of the hygiene textile product with these alternative material will appear to be bright and the demand for cost saving with high performance will fulfill by this research work.

\section{REFERENCES}

1. Keith J. Carnes, David A. Murillo, US 2004/ 0254551A1, (2004).

2. H. A. Saeed, Y. Liu, L. A. Lucia and H. Chen, BioResources., 12(2), 4166 (2017).

3. S. Björquist, J. Aronsson, G. Henriksson and A. Persson, Text. Res. J., 88(21), 2485 (2018).

4. M. Ansari, T. Eslaminejad, Z. Sarhadynejad and T. Eslaminejad, Eur. J. Med. Plants., 3(1), 135 (2013).

5. D. Michel, B. Bachelier, J. Y. Drean and O. Harzallah, Conference Papers in Science, (2013).

6. T. Theodore, C. Mozer, P. Joseph, J. Njie, N. Clins, Z. E. Parfait and S. J. Loic, Open. J. Appl. Sci., 10(8), 521 (2020).

7. E. Galiwango, N. S. A. Rahman, A. H. Al-Marzouqi, M. M. Abu-Omar and A. A. Khaleel, waste. Heliyon., 5(12), 1 (2019).

8. K. Olejnik, Fibers. Text. East. Euro., 20(1), 113 (2012). 